\title{
Three dimensional MRI estimates of brain and spinal cord atrophy in multiple sclerosis
}

\author{
Clarence Liu, Simon Edwards, Qiyong Gong, Neil Roberts, Lance D Blumhardt
}

\begin{abstract}
Objective-The association between brain atrophy and permanent functional deficits in multiple sclerosis and the temporal relation between atrophy and the clinical disease course have seldom been investigated. This study aims to determine the amount of infratentorial and supratentorial atrophy in patients by comparison with healthy controls, to establish the relation between atrophy and disability, and to derive the rates of volume loss in individual patients from their estimated disease durations.

Methods-Three dimensional acquired MRI was performed on 20 relapsingremitting and 20 secondary progressive multiple sclerosis patients and 10 control subjects. Volume data on infratentorial and supratentorial structures were obtained using the Cavalieri method of modern design stereology in combination with point counting. Corpus callosal sectional area and "T2 lesion load" were also determined.
\end{abstract}

Results-Significantly reduced infratentorial and cerebral white matter volumes and corpus callosal sectional areas occurred in all patients compared with controls $(p=0.0001-0.004)$. Mean estimates of volume loss in the cohort were $-21 \%,-19 \%,-46 \%$, and $-12 \%$ for the brain stem, cerebellum, upper cervical cord and white matter, respectively, and $-21 \%$ for the corpus callosal sectional area. Analysis of the amount of atrophy (volume differences between patients and controls) showed that upper cervical cord and cerebral white matter atrophy correlated with the expanded disability status scale $(r=-0.37$ and $-0.37, p=0.018-0.023)$ and the Scripps neurologic rating scale scores $(r=+0.49$ and $+0.43, p=0.002-0.007)$. There was no relation between estimated volume loss in the supratentorial and infratentorial compartments. The "T2 lesion load" was associated with ventricular enlargement and corpus callosal atrophy $(r=+0.50$ and $-0.55, p=0.0003-0.0012)$. Infratentorial atrophy rates correlated with baseline exacerbation rates $(r=-0.50$ to -0.48 , $\mathrm{p}=0.0016-0.0021)$ and were higher in relapsing-remitting than secondary progressive patients $(p=0.009-0.02)$.

Conclusions-Significant cerebral and spinal cord volume reductions occurred in both patient subgroups compared with controls. Functional correlates were found with estimated volume loss in the upper cervical cord and cerebral white matter. Particularly for infratentorial structures, estimated rates of atrophy were higher in relapsing-remitting than secondary progressive patients, suggesting that atrophy, perhaps mainly due to tract degeneration, begins early in multiple sclerosis and may relate predominantly to acute inflammatory events, with or without other gradual non-inflammatory processes later in the disease course.

(f Neurol Neurosurg Psychiatry 1999;66:323-330)

Keywords: multiple sclerosis; atrophy; 3-D MRI

Relapsing-remitting (RR) and secondary progressive (SP) multiple sclerosis (MS) are widely accepted as different phases of the same disease. ${ }^{12}$ After an initial period of relapses and remissions, about $50 \%$ to $60 \%$ of RR patients will convert to the chronic progressive stage within 10 years of onset. ${ }^{3}{ }^{4}$ The pathophysiological basis for this change is uncertain, although it has long been supposed that whereas inflammation and demyelination predominate in early RR MS, axonal loss and subsequent atrophy are late events, associated with chronic lesions and the development of irreversible neurological deficits in the SP phase. ${ }^{5}$ In addition, axonal loss is thought to be a gradual process with the passage of time. ${ }^{6}$ These ideas have been challenged by the demonstration that axonal loss in the form of defects in the retinal nerve fibre layer is surprisingly common in optic neuritis. ${ }^{7}$ Further, histopathological series have disclosed that substantial axonal loss occurs in acute MS plaques, ${ }^{8}$ even when they are clinically silent. ${ }^{9}$ Magnetic resonance spectroscopy has been increasingly employed in the investigation of chemical changes in MS patients, with reduction in N-acetyl aspartate (NAA) peaks in both MS lesions and normal appearing white matter being taken to represent axonal loss. ${ }^{10-14}$ None the less, there is uncertainty as to whether

Table 1 Profile of patients (medians (interquartile range))

\begin{tabular}{lll}
\hline & $R R$ & $S P$ \\
\hline Number of patients & 20 & 20 \\
Female : Male & $14: 6$ & $13: 7$ \\
Age & $32(26-39)^{\star}$ & $40(34-46)$ \\
Disease duration & $4(3-7)^{\star \star}$ & $11(7-16)$ \\
Relapse rate in 2 y & $3(3-5)^{\star \star}$ & $1(0-2)$ \\
EDSS & $2.0(1.0-2.5)^{\star \star}$ & $6.0(5.0-6.0)$ \\
AI & $1(0-1)^{\star \star}$ & $4(3-4)$ \\
SNRS & $94(87-98)^{\star \star}$ & $72(63-74)$ \\
\hline
\end{tabular}

EDSS=Expanded disability status scale; $\mathrm{AI}=$ ambulation index; SNRS=Scripps neurologic rating scale; All comparisons significant $\left({ }^{\star} \mathrm{p} 0.01 ;{ }^{\star \star} \mathrm{p}<0.001\right)$. 
Table 2 Mean (SD) normalised volumes $(\mathrm{ml})$ for control subjects and all patients and mean (SD) volume changest ( $\mathrm{ml}$ and \%) for all patients

\begin{tabular}{lcccc}
\hline & \multicolumn{1}{l}{ Control } & \multicolumn{1}{l}{ All } & \multicolumn{1}{l}{ p Value } & \multicolumn{1}{c}{ Volume changet } \\
\hline Brain stem & $28.1(2.4)$ & $22.3(3.1)$ & $<0.0001^{\star \star}$ & $-5.8(3.1)-20.6 \%$ \\
Cerebellum & $147.8(11.0)$ & $120.1(13.7)$ & $0.0001^{\star \star}$ & $-27.6(13.7)-18.7 \%$ \\
Upper cervical cord & $4.4(0.68)$ & $2.4(0.68)$ & $<0.0001^{\star \star}$ & $-2.1(0.68)-46.4 \%$ \\
Cerebral grey matter & $590.3(47.2)$ & $590.8(53.5)$ & NS & $+0.5(53.5)+0.1 \%$ \\
Cerebral white matter & $465.5(44.0)$ & $409.9(47.1)$ & $0.0007^{\star \star}$ & $-55.6(47.1)-11.9 \%$ \\
Cerebral hemispheres & $1055.8(80.9)$ & $1000.1(65.0)$ & $0.047^{\star}$ & $-56.0(65.0)-5.3 \%$ \\
Ventricles & $14.8(10.0)$ & $17.9(11.3)$ & NS & $+3.2(11.3)+21.3 \%$ \\
Corpus callosum $\ddagger$ & $6.4(1.4)$ & $5.1(1.0)$ & $0.004^{\star \star}$ & $-1.4(1.0)-21.4 \%$ \\
\hline
\end{tabular}

tMean of (individual patient volume - mean control volume); $\neq$ Corpus callosal area in $\mathrm{cm}^{2} ; \star$ Trend $(0.006<\mathrm{p}<0.05) ;{ }^{\star \star}$ significant after Bonferroni $(\mathrm{p}<0.006)$.

reductions in NAA correspond to permanent or merely transient axonal dysfunction. ${ }^{14}$ Another possibility is to investigate loss of tissue volume, which, although lacking in specificity for demyelination or axonal loss, is correlated with irreversible neurological dysfunction. ${ }^{15-22}$

In this study, we acquired high resolution three dimensional (3-D) T1 weighted gradient echo MR images of the brain ${ }^{23}$ in a cohort of $\mathrm{RR}$ and SP MS patients as well as control subjects and employed the Cavalieri method of modern design stereology in combination with point counting to obtain unbiased estimates of structural volumes. ${ }^{24}$ Our aims were to determine, firstly, how much atrophy of the brain and spinal cord occurs in RR and SP patients compared with controls; secondly, the functional relevance of atrophy, and thirdly, the relative rates of volume loss in RR and SP MS.

\section{Methods}

SUBJECTS

Forty patients with clinically definite or laboratory supported definite $M S^{25}$ were recruited. Twenty were classified as RR and 20 as SP $\mathrm{MS}^{26}$ The disease course for each patient, including the initial episode and subsequent relapses and progress, was established by reviewing the clinical histories and neurological records. All the RR patients had at least two documented relapses, as defined by the Poser criteria, ${ }^{25}$ in the 2 years before the study, and had been free of exacerbations for at least 3 months. No patient had been treated with immunosuppressive drugs or immunomodulators and none had any other relevant medical conditions. Full neurological examinations were performed on the cohort within 24 hours of MRI. Disability was scored on the expanded disability status scale (EDSS), ${ }^{27}$ the ambulation index,${ }^{28}$ and the Scripps neurologic rating scale (SNRS). ${ }^{29}$ Ten healthy volunteers (five men and five women), age matched to the RR subgroup, also underwent MRI.

MRI PROTOCOL

Brain MRI was performed on a $1.5 \mathrm{~T}$ system (Magnetom SP4000, Siemens, Erlangen) with a proprietary head coil. A proton density and T2 weighted dual echo sequence with $5 \mathrm{~mm}$ transverse contiguous scans was acquired (TR $2500 \mathrm{~ms}$; TE(PD) $27 \mathrm{~ms}$, TE(T2-W) $81 \mathrm{~ms}$; matrix 192×256; FOV $25 \mathrm{~cm}$ ). Ten minutes after intravenous gadolinium-DPTA at 0.1 $\mathrm{mmol} / \mathrm{kg}$, a T1 weighted 3-D gradient echo sequence (magnetisation prepared rapid acquisition gradient echo: MP-RAGE) was obtained (TR 10 ms; TE 4 ms; TD 100 ms; TI 300 ms; flip angle $10^{\circ}$; matrix $192 \times 256$; FOV $25 \mathrm{~cm}$ ). This provided 128 sagittal images with an effective slice thickness of $1.4 \mathrm{~mm}$.
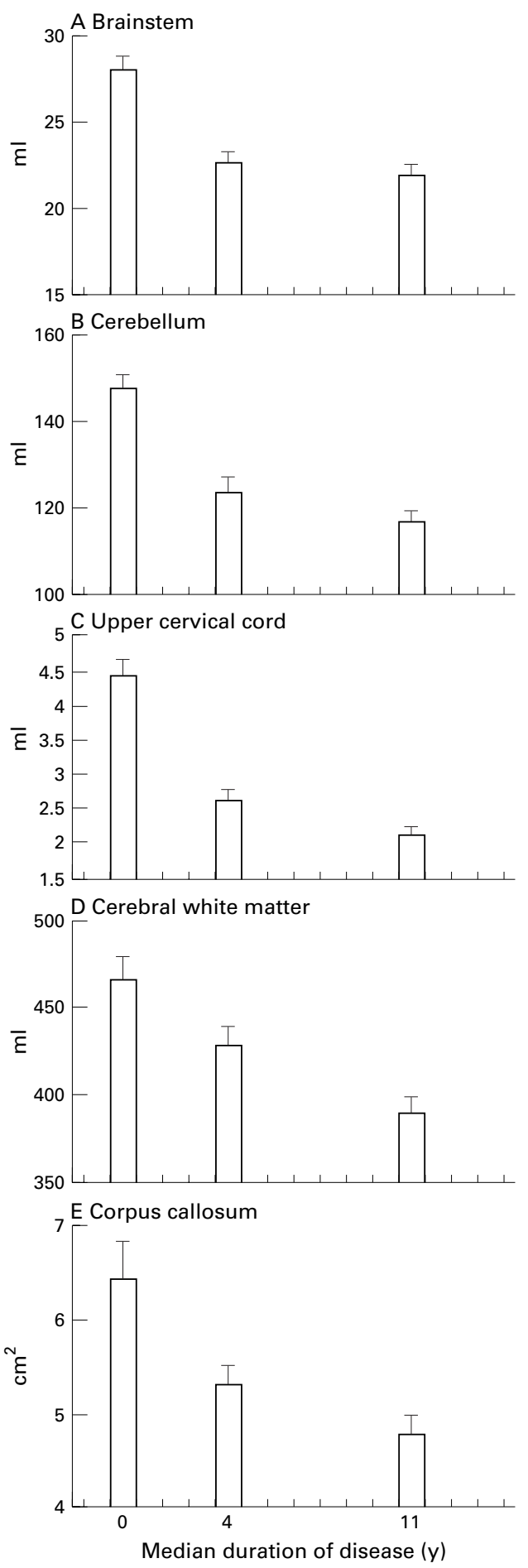

Figure 1 Mean normalised volumes (with SEM) for $(A)$ brain stem, (B) cerebellum, (C) upper cervical cord, (D) cerebral white matter, and (E) mean corpus callosal area, plotted v median disease duration (from initial neurological episode). In each graph, the first column represents the controls (assuming the control group at year 0), the second, $R R$ patients, and the third, SP patients. 
Table 3 Mean (SD) normalised volumes $(\mathrm{ml})$ and mean (SD) volume changest $(\mathrm{ml}$ and \%) of RR and SP patients

\begin{tabular}{|c|c|c|c|c|c|c|}
\hline & $R R$ & $S P$ & $p(1)$ & $R R$ volume change $\neq$ & SP volume change $\neq$ & p Value (2) \\
\hline Brain stem & $22.6(2.9)$ & $21.9(3.3)$ & NS & $-5.4(2.9)-19.3 \%$ & $-6.1(3.3)-21.8 \%$ & NS \\
\hline Cerebellum & $123.6(2.9)$ & $116.7(11.1)$ & NS & $-24.2(15.4)-16.4 \%$ & $-31.1(11.1)-21.0 \%$ & NS \\
\hline Upper cervical cord & $2.6(0.68)$ & $2.1(0.59)$ & $0.01^{\star}$ & $-1.8(0.68)-40.8 \%$ & $-2.3(0.59)-52.0 \%$ & $0.01^{\star}$ \\
\hline Cerebral grey matter & $582.0(39.1)$ & $599.7(64.7)$ & NS & $-8.3(39.1)-1.4 \%$ & $+9.4(64.7)+1.6 \%$ & NS \\
\hline Cerebral white matter & $429.5(45.1)$ & $390.4(41.4)$ & $0.007^{\star}$ & $-36.0(45.1)-7.7 \%$ & $-75.2(41.4)-16.2 \%$ & $0.008^{\star}$ \\
\hline Cerebral hemispheres & $1010.2(58.7)$ & $990.0(70.9)$ & NS & $-45.6(58.7)-4.3 \%$ & $-65.9(70.9)-6.2 \%$ & NS \\
\hline Ventricles & $16.1(13.2)$ & $19.8(9.0)$ & NS & $+1.3(13.2)+8.8 \%$ & $+5.0(9.0)+33.8 \%$ & NS \\
\hline Corpus callosum $\ddagger$ & $5.3(1.1)$ & $4.8(1.0)$ & NS & $-1.1(1.1)-17.4 \%$ & $-1.6(1.0)-25.5 \%$ & NS \\
\hline
\end{tabular}

†Mean of (individual patient volume-mean control volume); $\neq$ Corpus callosal area in $\mathrm{cm}^{2} ; \mathrm{p}(1)=$ Comparison of normalised volumes for RR and SP; $p$ Value $(2)=$ comparison of volume changes for RR and SP; ${ }^{\star}$ Trend $(0.006<\mathrm{p}<0.05)$; ${ }^{\star \star}$ significant after Bonferroni $(\mathrm{p}<0.006)$.

MRI ANALYSIS

The MRI analysis was performed using ANALYZE software (Biomedical Imaging Resource, Mayo Foundation, MN, USA). Total intracranial volume (TICV) and volume estimates for the supratentorial (cerebral hemispheres, cerebral grey matter, cerebral white matter, and total ventricles (lateral, third, and fourth)) and infratentorial compartments (brain stem, cerebellum, upper cervical cord (C1-3)), were obtained from the 3-D MPRAGE images. The area of the corpus callosum was measured in the mid-sagittal plane as defined by the slice which included the full extent of the aqueduct. Structural boundaries were determined by reference to established landmarks. ${ }^{30}$

The Cavalieri method provides unbiased volume estimates. A test system, comprising a square array of grid points, is overlaid at a random orientation on a systematic series of sections, exhaustively sampling the structure of interest. The number of points covering the structural transects is recorded. The volumes are estimated as the sum of the points counted on all the transects multiplied by the sectioning intervals. ${ }^{24} 31$ To correct for variations in head size, all the volumes were normalised by the subject/population TICV ratio. ${ }^{32}$

The total brain lesion volume on the T2 weighted scans (T2 lesion load) in each patient was assessed with a seed growing and signal intensity thresholding technique ${ }^{33}$ using ANALYZE software.

DATA ANALYSIS

Individual normalised volume measurements for each patient were subtracted from the corresponding mean control data to obtain the volume differences (in $\mathrm{ml}$ and \%). The rates of atrophy (in $\mathrm{ml} /$ year and \% change/year) were then estimated with reference to each patient's disease duration (time from onset of the first neurological episode or relapse).

Statistical analysis was performed using Arcus Pro-II software (version 2.15). Clinical differences were assessed with the MannWhitney $U$ test (two tailed). Rater and scanning variabilities were calculated according to the method of Bland and Altman. ${ }^{34}$ Intergroup comparisons of MRI data were made with the Kruskal-Wallis test. Correlations were explored with Spearman's rank coefficient. Means, SD, and SEM were calculated when data were normally distributed (Shapiro-Wilk $W$ test).

Due to the relatively large number of tests carried out, a Bonferroni procedure was performed on each set of comparisons to estimate the $\mathrm{p}$ values required for significance. ${ }^{35}$

\section{Results}

Our MS patient cohort (13 men, 27 women) had a median age of 36 (interquartile range (IQR) 29 to 44) with a median disease duration of 7 years (IQR 4 to 13) and a median baseline 2 year exacerbation rate of 3 (IQR 1 to 4 ). The median neurological rating scores for EDSS, ambulation index, and SNRS were 3.5, 2 and 78 , respectively. The controls comprised five men and five women whose age (median 30, IQR 26 to 32) did not differ significantly from the RR subgroup. The SP patients were significantly older than their RR counterparts and had longer disease durations, lower relapse rates, and worse scores on the clinical rating scales (table 1). There was no subgroup difference in height.

The estimated T2 lesion loads for all patients had a median of $18.9 \mathrm{~cm}^{2}$ (IQR 7.1 to 32.7 ), and the RR (median $16.1 \mathrm{~cm}^{2}$, IQR 6.5 to 28.1) and SP (median $22.3 \mathrm{~cm}^{2}$, IQR 7.8 to 36.8) T2 lesion loads were not significantly different.

For the volume estimates, within and between operator variance on 10 sets of randomly selected scans ranged from 0.02 to 0.03 and 0.05 to 0.06 , respectively. The scan-rescan variability from phantom volume measurements was 0.02 . There were no significant between group differences in TICV.

Table 4 Relation between structural volume changes (normalised volume differences between patients and controls) (Spearman's rank correlation coefficients)

\begin{tabular}{|c|c|c|c|c|c|c|c|}
\hline \multicolumn{8}{|l|}{ Brain stem } \\
\hline$+0.52^{\star \star} \mathrm{p}=0.0011$ & NS & $U C C$ & & & & & \\
\hline NS & NS & NS & Grey matter & & & & \\
\hline NS & $+0.34^{\star} \mathrm{p}=0.034$ & NS & NS & White matter & & & \\
\hline NS & $+0.36^{\star} \mathrm{p}=0.024$ & NS & $+0.61^{\star \star} \mathrm{p}<0.0001$ & $+0.55^{\star \star} \mathrm{p}=0.0003$ & Cerebral hemisphere & & \\
\hline NS & $-0.31^{\star} \mathrm{p}=0.050$ & NS & NS & $-0.39^{\star} p=0.013$ & $-0.54^{\star \star} p=0.0004$ & Ventricles & \\
\hline NS & $+0.39^{\star} \mathrm{p}=0.013$ & NS & NS & $+0.57^{\star \star} \mathrm{p}=0.0002$ & $+0.69^{\star \star} \mathrm{p}<0.0001$ & $-0.65^{\star \star} p<0.0001$ & Corpus callosum \\
\hline
\end{tabular}

Shaded areas denote comparisons between infratentorial and supratentorial structures; UCC=upper cervical cord.

*Trend $(0.002<\mathrm{p}<0.05)$; ${ }^{\star \star}$ significant after Bonferroni $(\mathrm{p}<0.002)$. 
Table 5 Correlations between structural volume changes (normalised volume differences between patients and controls) and clinical data (Spearman's rank correlation coefficients)

\begin{tabular}{lll}
\hline & Upper cervical cord & Cerebral white matter \\
\hline EDSS & $-0.37(\mathrm{p}=0.023)^{\star}$ & $-0.37(\mathrm{p}=0.018)^{\star}$ \\
AI & $-0.33(\mathrm{p}=0.045)^{\star}$ & $-0.35(\mathrm{p}=0.027)^{\star}$ \\
SNRS & $+0.49(\mathrm{p}=0.002)^{\star \star}$ & $+0.43(\mathrm{p}=0.007)^{\star}$ \\
Disease duration & $-0.38(\mathrm{p}=0.019)^{\star}$ & $-0.45(\mathrm{p}=0.004)^{\star \star}$ \\
Exacerbation rate (in last 2 y) & $+0.34(\mathrm{p}=0.040)^{\star}$ & $+0.33(\mathrm{p}=0.036)^{\star}$ \\
\hline
\end{tabular}

*Trend $(0.005<\mathrm{p}<0.05) ;{ }^{\star \star}$ Significant after Bonferroni $(\mathrm{p}<0.005)$.
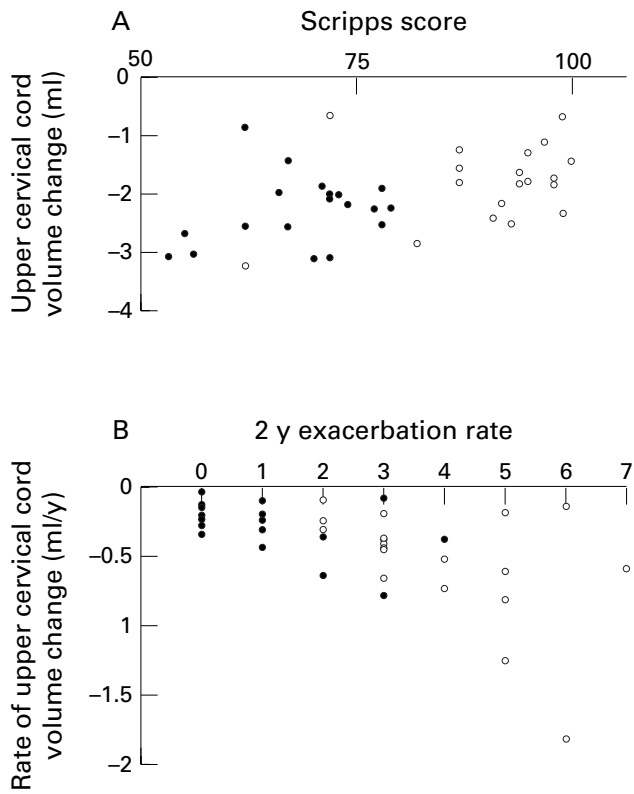

Figure 2 Scatter plots of $(A)$ normalised volume change in the upper cervical cord $v$ Scripps neurologic rating scale score and $(B)$ rate of volume of change in the upper cervical cord $v 2$ year exacerbation rate, for RR (open circles) and SP (filled circles) patients. The Spearman's rank correlation coefficients $(r)$ are $(A),+0.49(p=0.002)$ and $(B),-0.50(p=0.002)$.

The control subjects had significantly larger infratentorial and cerebral white matter volumes and corpus callosal areas than either patient subgroups ( $\mathrm{p}<0.0001$ to 0.004 ) (table 2 , fig 1). These results correspond to mean volume changes in the brain stem of $-21 \%$, the cerebellum of $-19 \%$, the upper cervical cord of $-46 \%$, and the cerebral white matter of $-12 \%$.

Table 6 Rates of volume changet derived from disease duration. [Mean rates (SD) of change (ml/year) and mean \% change in RR/SP patients]

\begin{tabular}{|c|c|c|c|}
\hline & $R R$ change & SP change & $p$ Value \\
\hline Brain stem & $-2.0(2.1)-6.9 \% / y$ & $-0.7(0.6)-2.5 \% / y$ & $0.02^{\star}$ \\
\hline Cerebellum & $-9.3(11.4)-6.3 \% / y$ & $-3.5(2.3)-2.3 \% / \mathrm{y}$ & NS \\
\hline Upper cervical cord & $-0.54(0.41)-12.2 \% / y$ & $-0.27(0.19)-6.1 \% / y$ & $0.009^{\star}$ \\
\hline Cerebral grey matter & $-3.8(23.9)-0.6 \% / y$ & $-1.4 \quad(8.5)-0.2 \% / y$ & NS \\
\hline Cerebral white matter & $-9.5(16.6)-2.0 \% / \mathrm{y}$ & $-8.7(8.2)-1.9 \% / \mathrm{y}$ & NS \\
\hline Cerebral hemispheres & $-14.5(33.8)-1.4 \% / y$ & $-7.3(8.7)-0.7 \% / y$ & NS \\
\hline Ventricles & $-0.1 \quad(6.2)-0.9 \% / y$ & $+0.6 \quad(1.1)+3.8 \% / y$ & NS \\
\hline Corpus callosum $\ddagger$ & $-0.3(0.46)-5.3 \% / y$ & $-0.2(0.16)-2.8 \% / y$ & NS \\
\hline
\end{tabular}

†Volume changes derived from table 3 ; $¥$ Corpus callosal area in $\mathrm{cm}^{2}$.

${ }^{\star}$ Trend $(0.006<p<0.05) ;{ }^{\star \star}$ significant after Bonferroni $(\mathrm{p}<0.006)$.

Table 7 Correlations between extrapolated rates of infratentorial volume change and clinical data (Spearman's rank correlation coefficients)

\begin{tabular}{llll}
\hline & Brain stem & Cerebellum & Upper cervical cord \\
\hline EDSS & $+0.34(\mathrm{p}=0.03)^{\star}$ & $\mathrm{NS}$ & $+0.36(\mathrm{p}=0.03)^{\star}$ \\
AI & $+0.42(\mathrm{p}=0.007)^{\star}$ & $+0.34(\mathrm{p}=0.03)^{\star}$ & $+0.46(\mathrm{p}=0.004)^{\star}$ \\
SNRS & $\mathrm{NS}$ & $\mathrm{NS}$ & $\mathrm{NS}$ \\
$\begin{array}{l}\text { Exacerbation rate } \\
\quad \text { in past 2 y) }\end{array}$ & $-0.48(\mathrm{p}=0.0021)^{\star \star}$ & $-0.42(\mathrm{p}=0.0082)^{\star}$ & $-0.50(\mathrm{p}=0.0016)^{\star \star}$
\end{tabular}

${ }^{\star}$ Trend $(0.004<\mathrm{p}<0.05) ;{ }^{\star \star}$ significant after Bonferroni $(\mathrm{p}<0.004)$.
The mean change in cross sectional area of the corpus callosum was $-21 \%$ (table 2). A comparison of the patient subgroups showed trends to more atrophy of the cerebral white matter and upper cervical cord in SP than RR MS ( $p=0.01$ to 0.008 ) (table 3).

No significant association was found between supratentorial and infratentorial volume losses, although trends were seen between cerebellar atrophy and tissue loss in the white matter, cerebral hemispheres, and corpus callosum ( $r=+0.34$ to +0.39 ; $\mathrm{p}=0.013$ to 0.034 ), as well as increase in ventricular size $(r=-0.31 ; \mathrm{p}=0.05)$ (table 4). The T2 lesion load correlated significantly with both an increase in ventricular size $(r=+0.50 ; \mathrm{p}=0.0012)$ and a reduction in corpus callosal area $(r=-0.55 ; \mathrm{p}=0.0003)$, but not with the volumes of the cerebral white matter or infratentorial structures.

For the entire patient cohort, the volume change in the upper cervical cord (difference from control values) correlated significantly with disability, such that a reduction in SNRS was associated with more atrophy $(r=+0.49$; $\mathrm{p}=0.002$ ) (table 5, fig $2 \mathrm{~A}$ ). Supratentorial loss in white matter volume (difference from controls) was correlated significantly with disease duration $(r=-0.45 ; \mathrm{p}=0.004)$ and followed a trend such that the lower the SNRS, the greater the reduction in white matter volume $(r=+0.43 ; \mathrm{p}=0.007)$ (table 5$)$. Trends were also seen for associations between increases in EDSS and ambulation index and volume reductions in both the upper cervical cord and cerebral white matter $(r=-0.33$ to -0.37 ; $\mathrm{p}=0.018$ to 0.045 ). The T2 lesion load did not correlate with any clinical rating scores.

Rates of supratentorial volume changes, as derived from individual disease durations, showed no significant subgroup differences (table 6). Infratentorially, the upper cervical cord and brain stem volumes in the RR patients seemed to have declined at a faster rate than for the SP subgroup (table 6). For the entire cohort, the atrophy rates of the upper cervical cord and brain stem also correlated significantly with the 2 year relapse rates $(r=-0.50$, -0.48; $\mathrm{p}=0.0016$ to 0.0021 ) - that is, the higher the baseline relapse rate, the faster the volume loss (table 7, fig $2 \mathrm{~B}$ ). Infratentorial atrophy rates also correlated positively with increases in EDSS and ambulation index $(r=+0.34$ to $+0.46 ; \mathrm{p}=0.004$ to 0.03 ) (table 7$)$.

\section{Discussion}

SUPRATENTORIAL ATROPHY

Our MRI results showed significant atrophy of both cerebral white matter and the corpus callosum, as well as a trend to greater volume loss of the cerebral white matter in SP compared with RR MS. In our patients, cerebral white matter atrophy (on average $12 \%$ less than controls) was correlated with the SNRS and the EDSS scores, suggesting that white matter volume loss may have functional significance. On the other hand, our T2 lesion load measurements did not show clinical correlations. This should not be surprising, given that the average T2 lesion load, when extrapolated to 3-D data, represents less than $4 \%$ of the white matter 
volume in our cohort. Recent MR spectroscopic evidence suggests that a far greater proportion of the reduction in brain NAA is contributed by changes in the normal appearing white matter than by the total $\mathrm{T} 2$ weighted lesions. ${ }^{13}$ It is thus intriguing to consider whether cerebral white matter atrophy may be more strongly associated with the invisible lesion load in the normal appearing white matter, ${ }^{36}$ than with lesions visible on $\mathrm{T} 2$ weighted images.

In some previously published series it is possible to estimate the amount of atrophy of the corpus callosum from the data provided. Using the control data from the series of Pelletier et al, the mean loss of tissue in the corpus callosum on the mid-sagittal slice can be estimated as $25 \%$ and $15 \%$, for their patients with and without T2 weighted brain lesions, respectively. ${ }^{37}$ Similarly, utilising MRI measurements from the study of Simon et al, a mean loss of $18 \%$ and $11 \%$ of corpus callosal area can be derived for their male and female patients, respectively. ${ }^{38}$ These results compare with a mean loss of $21 \%$ in our overall cohort. Our findings of a significant association between corpus callosal atrophy and the T2 lesion load are consistent with these earlier studies. The lack of correlations of our data with clinical disability may be explained partly by the detection of a substantial reduction in axonal density in additional to atrophy in the normal appearing white matter of the corpus callosum from a recent histopathological report. ${ }^{39}$

There were trends to cerebral atrophy and ventricular enlargement in our patients, but there are few publications on cerebral atrophy in MS with which to compare our results. In one study of longitudinal volume changes in MS using 4 slice MRI "slabs", no controls were examined. ${ }^{17}$ The failure of our ventricular comparisons to reach significance might be confounded by the relatively large variance in the data and possible fluctuations of ventricular size with the state of hydration. ${ }^{40} 41$ In addition, the method of volume normalisation described by Blatter et al fails to correct for age and sex differences in the ventricular size of healthy subjects. ${ }^{32}$

INFRATENTORIAL ATROPHY

Our patients had significantly smaller volumes than healthy controls in all three infratentorial structures. Differences between RR and SP MS were apparent for the upper cervical cord. Previous reports have mainly focused on measurements of the cerebellum and the cervical cord. For the cerebellum, earlier series concentrated on lesion load, ${ }^{42}$ although more recently Davie et al compared the cerebellar volumes of MS patients with (Kurtzke cerebellar function score $\geqslant 3$ ) and without ataxia (cerebellar score $0-1) .{ }^{43}$ The amount of cerebellar atrophy can be estimated from their control data (bearing in mind that only the median control volume is available), as loss of $15 \%$ and $5 \%$ for the ataxic and non-ataxic patients, respectively. Although direct comparison with our study would not be strictly accurate due to confounding factors from dissimilar case mix (both our RR and SP subgroups had cerebellar scores $0-3$ ) as well as different scanning (2-D $v 3-\mathrm{D}$ acquired MRI) and analytical techniques (contouring $v$ stereology; raw $v$ normalised volumes), our cohort showed a higher cerebellar volume loss than that of Davie et al. However, the functional relevance of this is unclear as our results did not correlate with any clinical indices, including the cerebellar function score.

The cross sectional area of the cervical cord at $\mathrm{C} 5$ has been investigated in several series with conventional 2-D MRI. ${ }^{15}{ }^{16}{ }^{44-46}$ If extrapolation is performed from the available control data in the study of Kidd et al, a mean reduction in area of $9 \%$ can be derived in both $\mathrm{RR}$ and SP subgroups (with less atrophy in their primary progressive and benign patients). ${ }^{15}$ This compares with a mean decrease of $16 \%$ in SP MS from another report. ${ }^{16}$ The cord area at C2 has also been examined using MR techniques with higher reproducibility. Losseff et al measured C2 area with a volume acquired inversion prepared sequence. ${ }^{18}$ From their control data there was no significant area loss in RR patients, whereas their SP subgroup sustained a mean loss of $28 \%$ (the last having more atrophy than their primary progressive and benign counterparts in the study). Again, methodological issues, especially between 2-D and 3-D data, confound direct comparisons. Nevertheless, the relatively high amount of cervical atrophy in the present study correlated significantly with the SNRS and conferred trends for the EDSS and ambulation index, thus suggesting the relevance of volume loss to functional deficits in the pathway rich infratentorial region.

RELATION OF STRUCTURAL CHANGES

From our demonstration of both supratentorial and infratentorial atrophy, some spinal cord volume loss might be expected secondary to changes "upstream" in the cerebral hemispheres due to Wallerian degeneration. ${ }^{47-49}$ In our study, despite the association between reductions in structural volume within each intracranial compartment, no significant relation was found between supratentorial and infratentorial atrophy (table 4). Furthermore, shrinkage of the upper cervical cord and the cerebral white matter did not correlate with T2 lesion load. Hence the volume changes in the spinal cord seem to occur independently of brain abnormalities, in agreement with the conclusion from another series. ${ }^{21}$ None the less we cannot exclude the possibility of other indices (for example, cervical cord T2 and T1 lesion loads) being related to supratentorial changes. Interestingly, our T2 lesion load, although not associated significantly with increased disability, correlated well with corpus callosal atrophy and ventricular enlargement. The importance of this perhaps lies in possible adverse consequences on cognition. Moreover, it has been suggested that T2 weighted lesions may be a prerequisite for the development of atrophy in the corpus callosum, ${ }^{37}$ especially relating to axonal loss secondary to periventricular plaques. ${ }^{38}$ It has also been reported in a postmortem study that greater atrophy of the 
corpus callosum is associated with more marked ventricular dilatation..$^{50}$

ATROPHY RATES

The inevitable uncertainty about the reliability of retrospective data based on the time from the first episode of neurological symptoms, means that cautious interpretation of our atrophy rate estimates is necessary, especially in view of the lack of information about the duration of the "latent phase" before clinical manifestations of MS. ${ }^{6}$ However, as RR and SP MS have long been regarded as different phases of the same disease, ${ }^{12}$ and up to $60 \%$ of $\mathrm{RR}$ patients are expected to become progressive by 10 years, ${ }^{3451}$ it is instructive to consider our subgroup results as an approximation of the volume changes from no clinical disease through RR MS to SP MS. Whereas the projected supratentorial structural volume loss would seem to remain constant over time, the rates of infratentorial atrophy appear to decrease with longer duration. Indeed our RR brain stem and upper cervical cord atrophy rates followed a trend to be greater than those of the SP patients (table 6).

The above findings are unexpected, as it has been generally accepted that pathologically early MS consists of mainly inflammation and demyelination, whereas severe axonal loss and atrophy are events of chronic or late $\mathrm{MS}^{5}$ Axonal destruction has also been thought to occur gradually and progressively throughout the disease course. ${ }^{6}$ However, several recent experimental, neuroimaging, and neuropathological series have suggested otherwise. Firstly, using Theiler's murine encephalomyelitis virus as an experimental model of MS in transgenic mice, Rivera-Quinones et al demonstrated that in class I major histocompatibility complex deficient mice with severe demyelination, but preserved axons in the spinal cord, little functional deficit was seen. However, in other groups of mice with demyelinated spinal cords as well as axonal damage, profound neurological dysfunction ensued. ${ }^{52}$ Secondly, there is MR spectroscopic evidence that the rate of supratentorial NAA decline is consistently slower in SP than RR MS. ${ }^{12}$ The rate of axonal damage may be proportional to the density of existing axons, so that further inflammation may result in less axonal damage in the later stages of $\mathrm{MS} .^{13}$ Infratentorially, a serial MRI study of cervical cord area at C2 reported increasing atrophy over 1 year in RR patients, but no significant change in SP MS. ${ }^{53}$ Thirdly, abundant evidence of axonal transection was disclosed in a histopathological series of active and chronic active MS lesions, suggesting that axonal changes occur early in the disease. ${ }^{54}$ Another postmortem study of clinically silent MS showed axonal loss of up to $64 \%$ and $82 \%$ in demyelinated brain and spinal cord lesions, respectively. ${ }^{9}$ Pathological changes have also been found in the normal appearing white matter of the corpus callosum, with a $40 \%$ reduction in axonal density. ${ }^{39}$ Ferguson et al, employing immunostaining for amyloid precursor protein as a neuropathological marker of early axonal damage, showed expression in acute, but not chronic lesions. ${ }^{8}$ It has therefore been postulated that axonal damage is associated with relapses and inflammation. ${ }^{8}$ The last point is supported by our findings that the infratentorial atrophy rates correlated significantly with baseline relapse rates (table 7, fig 2 B). These examples have implications for our study of brain and spinal cord atrophy, as axonal loss is increasingly recognised as a major contributor to atrophy. ${ }^{22} 43$

Our estimation of atrophy rates was made by comparing the patients' volume data with those from a cohort of normal controls. Despite several potential confounding factors, we think that such comparisons are valid. Firstly, although the number of control subjects was relatively small, the variance in their volume data did not affect the highly significant differences in the patient data, even after the conservative Bonferroni $t$ test correction. Secondly, the age matching of the RR, but not the SP patients, to the controls might cause bias from possible supratentorial atrophy associated with normal aging. However, several normative brain imaging databases have demonstrated that significant atrophy of the cerebral hemispheres and grey matter, ${ }^{5-57}$ and reduction in the grey/white matter ratio ${ }^{58}$ and the intracranial white matter fraction, ${ }^{58}$ occur only after the fifth decade, which is outside the age range of our SP patients. Nevertheless, increasing ventricular dilatation has been documented from the second decade in male volunteers (after volume normalisation) $)^{32}$ and our ventricular data may therefore be less reliable in the SP subgroup. On the other hand, we aimed to investigate the approximate volume changes through the stages of no clinical disease via RR MS to SP MS (fig 1), so that accurate age matching is less critical. Thirdly, it has been reported that the cross sectional area of the spinal cord at C5 correlates with the height of control subjects. ${ }^{44}$ We found no significant differences in the height of our subgroups. Fourthly, the sex ratio in our patients was biased towards female patients, which might have resulted in smaller volumes. This potential error was corrected by normalising the data using the method described by Blatter et al, ${ }^{32}$ which eliminates such differences in cohorts of male and female subjects.

\section{Conclusion}

We have found significant atrophy of the infratentorial compartment, cerebral white matter volume, and corpus callosal area in our cohort of MS patients using 3-D MRI measurements. The estimated volume loss in the upper cervical cord and cerebral white matter was correlated with disability scores. Furthermore, the infratentorial rates of atrophy, as derived from disease durations, were lower in the SP than the RR subgroup. Disease activity detected on MRI is up to 10 times greater than clinical events. ${ }^{59}$ If loss of brain tissue in MS is a gradual, progressive process, myelin and/or axonal loss may have begun during the latent phase before the first clinical episode. However, if the rate of axonal loss is dependent on inflammation (as suggested by 
the correlation with relapses), or the amount of viable axons available, then the situation is more complex. To investigate the temporal relation of structural volumes and disease course, it is necessary to study patients before they develop clinical MS. An obvious group would be those with an isolated syndrome of the CNS suggestive of demyelination, similar to the subjects recruited for a recent brain and spinal cord MR study. ${ }^{60}$ Although measuring loss of tissue volume is not specific for the pathological mechanisms which underlie atrophy, and the roles of demyelination and gliosis remain unclear, it is increasingly evident that axonal loss is a major contributory factor to both atrophy and irreversible neurological disability. ${ }^{22}$ Techniques of MR which enable more specific pathological processes to be interpreted in vivo-for example, the concurrent use of MR spectroscopy and magnetisation transfer imaging, ${ }^{61}$ may help elucidate the relative contributions of the different tissue elements in the atrophy of MS.

1 Birley JL, Dudgeon L. A clinical and experimental contribution to the pathogenesis of disseminated sclerosis. Brain 1921;44:150-212

2 Matthews WB. Clinical aspects: course and prognosis. In Matthews WB, Compston A, Allen IV, et al, eds. McAlpine's multiple sclerosis. 2nd ed. Edinburgh: Churchill Livingstone, 1991:139-64.

3 Broman T, Andersen O, Bergmann L. Clinical studies on multiple sclerosis. 1. Presentation of an incidence material from Gothenburg. Acta Neurol Scand 1981;63:6-33.

4 Confavreux C, Aimard G, Devic M. Course and prognosis Confavreux C, Aimard G, Devic M. Course and prognosis
of multiple sclerosis assessed by the computerized data of multiple sclerosis assessed by the computerized
processing of 349 patients. Brain 1980;103:281-300.

processing of 349 patients. Brain 1980;103:281-300.
5 Lassmann H, Suchanek G, Ozawa K. Histopathology and the blood-cerebrospinal fluid barrier in multiple sclerosis. Ann Neurol 1994;36:S42-46.

6 McDonald WI, Miller DH, Barnes D. The pathological evolution of multiple sclerosis. Neuropathol Appl Neurobio 1992;18:319-34.

7 MacFayden DJ, Drance SM, Douglas GR, et al. The retinal nerve fiber layer, neuroretinal rim area, and visual evoked potentials in MS. Neurology 1988;38:1353-8.

8 Ferguson B, Matyszak MK, Esiri MM, et al. Axonal damage in acute multiple sclerosis lesions. Brain 1997;120:393-9.

9 Mews I, Bergmann M, Bunkowski S, et al. Oligodendrocyte and axon pathology in clinically silent multiple sclerosis lesions. Multiple Sclerosis 1998;4:55-62.

10 Matthews PM, Pioro E, Narayana S, et al. Assessment of lesion pathology in multiple sclerosis using quantitative MRI morphometry and magnetic resonance spectroscopy. Brain 1996;119:715-22.

11 Davie CA, Barker GJ, Thompson AJ, et al. 1-H magnetic resonance spectroscopy of chronic cerebral white matter lesions and normal appearing white matter in multiple sclerosis. F Neurol Neurosurg Psychiatry 1997;63:736-42.

12 Arnold DL, Wolinsky JS, Matthews PM, et al. The use of magnetic resonance spectroscopy in the evaluation of the natural history of multiple sclerosis. $\mathcal{F}$ Neurol Neurosurg Psychiatry 1998;64(suppl 1)S94-101.

13 Fu L, Matthews PM, De Stefano N, et al. Imaging axonal damage of normal-appearing white matter in multiple sclerosis. Brain 1998;121:103-13.

14 Narayana PA, Doyle TJ, Lai D, et al. Serial proton magnetic resonance spectroscopic imaging, contrast-enhanced magnetic resonance imaging, and quantitative lesion volumetry in multiple sclerosis. Ann Neurol 1998;43:56-71.

15 Kidd D, Thorpe JW, Thompson AJ, et al. Spinal cord MRI using multi-array coils and fast spin echo. II. Findings in using multi-array coils and fast spin echo. II.

16 Filippi M, Campi A, Colombo B, et al. A spinal cord MRI study of benign and secondary progressive multiple sclerostudy of benign and secondary
sis. $\mathcal{F}$ Neurol $1996 ; 243: 502-5$.

17 Losseff NA, Wang L, Lai HM, et al. Progressive cerebral atrophy in multiple sclerosis. A serial MRI study. Brain 1996;119:2009-19

18 Losseff NA, Webb SL, O'Riordan JI, et al. Spinal cord atrophy and disability in multiple sclerosis. A new reproducible and sensitive MRI method with potential to monitor disease progression. Brain 1996;119:701-8.

19 Edwards SGM, Gong Q, Roberts N, et al. MRI volumes of infratentorial structures correlate with disability measures in multiple sclerosis. $\mathcal{F}$ Neurol Neurosurg Psychiatry 1997;61: 214 .

20 Lycklama a Nijeholt GJ, Barkhof F, Scheltens P, et al. MR of the spinal cord in multiple sclerosis: relation to clinical subthe spinal cord in multiple sclerosis: relation to clinical

21 Lycklama a Nijeholt GJ, Van Walderveen MAA, Castelijn $\mathrm{JA}$, et al. Brain and spinal cord abnormalities in multiple sclerosis. Correlations between MRI parameters, clinical subtypes and symptoms. Brain 1998;121:687-97.

22 Losseff NA, Miller DH. Measures of brain and spinal cord atrophy in multiple sclerosis. $\mathcal{F}$ Neurol Neurosurg Psychiatry 1998;64(suppl 1):S102-5.

23 Filippi M, Yousry T, Horsfield MA, et al. A high-resolution three-dimensionally $\mathrm{T}_{1}$-weighted gradient echo sequence improves the detection of disease activity in multiple sclerosis. Ann Neurol 1996;40:901-7.

24 Roberts N, Garden AS, Cruz-Orive LM, et al. Estimation of fetal volume by magnetic resonance imaging and stereology. Br F Radiol 1994;67:1067-77.

25 Poser CM, Paty DW, Scheinberg L, et al. New diagnostic criteria for multiple sclerosis: guide

26 Lublin FD, Reingold SC, for the National Multiple Sclerosis Society (USA) Advisory Committee on Clinical Trials for New Agents in Multiple Sclerosis. Defining the clinical for New Agents in Multiple Sclerosis. Defining the clinical vey. Neurology 1996;46:907-11.

27 Kurtzke JF. Rating neurologic impairment in multiple sclerosis: an expanded disability status scale (EDSS). Neurology 1983;33:1444-52.

28 Hauser SL, Dawson DM, Lehrich JR, et al. Intensive immunosuppression in progressive multiple sclerosis. A randomized, three-arm study of high-dose intravenous cyclophosphamide, plasma exchange, and ACTH. N Engl f Med 1983;308:173-80.

29 Sipe JC, Knobler RL, Braheny SL, et al. A neurologic rating scale (NRS) for use in multiple sclerosis. Neurology 1984;34:1368-72.

30 Daniels D, Haughton V, Naidich T. Cranial and spinal magnetic resonance imaging. An atlas and guide. New York: Raven Press, 1987.

31 Gundersen HJG. Stereology: the fast lane between neuroanatomy and brain function- or still only a tightrope? Acta Neurol Scand 1992;S137:8-13.

32 Blatter DD, Bigler ED, Gale SD, et al. Quantitative olumetric analysis of brain MR: normative database spanning 5 decades of life. Am $\mathcal{F}$ Neuroradiol 1995;16:241-51.

33 Van Walderveen MAA, Barkhof F, Hommes OR, et al. Correlating MRI and clinical disease activity in multiple sclerosis: relevance of hypointense lesions on short-TR/ short-TE ( $\mathrm{T}_{1}$-weighted) spin-echo images. Neurology 1995; 45:1684-90.

34 Bland JM, Altman DG. Statistical methods for assessing greement between two methods of clinical assessment. Lancet 1986;i:307-10.

35 Zolman JF. Biostatistics. Experimental design and statistical inference. New York: Oxford University Press, 1993.

36 Barbosa S, Blumhardt LD, Roberts N, et al. Magnetic resonance relaxation time mapping in multiple sclerosis: normal appearing white matter and the invisible lesion load. Magn Reson Imaging 1994;12:33-42.

37 Pelletier J, Habib M, Lyon-Caen O, et al. Functional and magnetic resonance imaging correlates of callosal involvement in multiple sclerosis. Arch Neurol 1993;50:1077-82.

38 Simon JH, Schiffer RB, Rudick RA, et al. Quantitative determination of MS-induced corpus callosum atrophy in vivo using MR imaging. Am f Neuroradiol 1987;8:599-604.

39 Evangelou N, Esiri M, Palace J, et al. A quantitative pathological study of axonal loss in the corpus callosum in multiple sclerosis. Multiple Sclerosis 1998;4:287.

40 Mellanby AR, Reveley MA. Effects of acute dehydration on computerised tomographic assessment of cerebral density and ventricular volume. Lancet 1982;ii:874.

41 Ron MA, Acker W, Shaw GK, et al. Computerized tomography of the brain in chronic alcoholism. A survey and follow-up study. Brain 1982;105:497-514.

42 Baumhefner RW, Tourtellote WW, Syndulko K, et al. Quantitative multiple sclerosis plaque assessment with magnetic resonance imaging. Its correlation with clinical parameters, evoked potentials, and intra-blood-brain barrier IgG synthesis. Arch Neurol 1990;47:19-26.

33 Davie CA, Barker GJ, Webb S, et al. Persistent functional deficit in multiple sclerosis and autosomal dominant cerebellar ataxia is associated with axon loss. Brain 1995;118:1583-92. Erratum. Brain 1996;119:1415.

44 Thorpe JW, Kidd D, Kendall BE, et al. Spinal cord MRI using multi-array coils and fast spin echo. I: technical aspects and fin

45 Kidd D, Thorpe JW, Kendall BE, et al. MRI dynamics of brain and spinal cord in progressive multiple sclerosis. $\mathcal{F}$ Neurol Neurosurg Psychiatry 1996;60:15-19.

46 Thorpe JW, Kidd D, Moseley IF, et al. Serial gadoliniumenhanced MRI of the brain and spinal cord in early relapsing-remitting multiple sclerosis. Neurology 1996;46: $373-8$.

47 Lumsden CE. An outline of the pathology of multiple sclerosis. In: McAlpine D, Lumsden CE, Acheson ED, eds. Multiple sclerosis. A reappraisal. Edinburgh: Churchill Livingstone, 1972:314-21

48 Prineas JW. Pathology of multiple sclerosis. In: Cook SD ed. Handbook of multiple sclerosis. 2nd ed. New York: Marcel Dekker 1996;223-55.

49 Sawlani V, Gupta RK, Singh MK, et al. MRI demonstration of Wallerian degeneration in various intracranial lesions and its clinical implications. F Neurol Sci 1997;146:103-8.

50 Barnard RO, Triggs M. Corpus callosum in multiple sclerosis. $\mathcal{F}$ Neurol Neurosurg Psychiatry 1974;37:1259-64.

51 Runmarker B, Andersen O. Prognostic factors in a multiple sclerosis incidence cohort with twenty-five years of followup. Brain 1993;116:117-34. 
52 Rivera-Quinones C, McGavern D, Schmelzer JD, et al. Absence of neurological deficits following extensive demy-
elination in a class I-deficient murine model of multiple elination in a class I-deficient murine

53 Stevenson VL, Leary SM, Losseff NA, et al. Spinal cord atrophy and disability in MS. A longitudinal study. Neurology 1998; 51:234-8

54 Trapp BD, Peterson J, Ransohoff RM, et al. Axonal transection in the lesions of multiple sclerosis. N Engl f Med 1998; 338:278-85.

55 Miller AKH, Alston RL, Corsellis JAN. Variation with age in the volumes of grey and white matter in the cerebral hemispheres of man: Measurements with an image analyzer. Neuropathol Appl Neurobiol 1980;6:119-32.

56 Hatazawa J, Masatoshi I, Yamaura H, et al. Sex difference in brain atrophy during aging: a quantitative study with computed tomography. 7 Am Geriatr Soc 1982;30:235-9.

57 Schwartz M, Creasey H, Grady CL, et al. Computed tomo- graphic analysis of brain morphometrics in 30 healthy men, aged 21 to 81 years. Ann Neurol 1985;17:146-57.

58 Guttmann CRG, Jolesz FA, Kikinis R, et al. White matter changes with normal aging. Neurology 1998;50:972-8.

59 McDonald WI, Miller DH, Thompson AJ. Are magnetic resonance findings predictive of clinical outcome in therapeutic trials in multiple sclerosis? The dilemma of interferon-beta. Ann Neurol 1994;36:14-18.

60 O'Riordan JI, Losseff NA, Phatouros C, et al. Asymptomatic spinal cord lesions in clinically isolated optic nerve, brain stem, and spinal cord syndromes suggestive of demyelination. F Neurol Neurosurg Psychiatry 1998;64:353-7.

61 Lee MA, Blamire A, Ho K, et al. Asymmetry of N-acetyl aspartate (NAA) in the internal capsule of patients with multiple sclerosis correlates with lateralisation of motor impairment: a spectroscopic, clinical and electrophysiological study. $\mathcal{F}$ Neurol 1998;245:384.

\section{NEUROLOGICAL STAMP}

\section{Theodor Meynert (1833-92)}

The centre of Austrian neurology during the 19th century was in Vienna. Vienna at that time was at its academic peak with the presence of such men as Skoda, Billroth, and Rokitansky. Meynert was born in Dresden and became Professor of Neurology and Psychiatry in Vienna (187392) where he performed studies in neuroanatomy and had a masterly grasp of the structure of the nervous system. He demonstrated that nerve cells in the cortex were in five horizontal layers (1867). In his book (1868) he discussed the cerebral cortex, hippocampal formation, olfactory bulb, and visual radiation. Eponymously he is remembered in Meynert's bundle, Meynert's commisure, the basal nucleus of Meynert, and the solitary cells of Meynert which are located in the region of the calcarine fissure. $\mathrm{He}$ singled out the calcarine fissure as being the most important of the occipital lobe. Meynert suggested that in addition to the occipital lobe, the whole temporal lobe may be involved in vision and that Parkinson's disease may be the result of defective function of the basal ganglia (1871). Among his contribution to the medical literature was Diseases of the Forebrain (1884). In that book he wrote on insanity as being a disease of the forebrain. He was editor of Fahrbucher Fur Psychiatrie from 1889 to 1892.

Meynert had a profound influence on the succeeding generation of neurologists including Sachs, Starr, and Putnam in America, and van Strumpell and Wernicke in Germany. Arnold Pick, of Pick's disease, who became Professor of Psychiatry in Prague, was also a student of Meynert, and Freud, in 1882, entered the General Hospital in Vienna as a clinical assistant to train with him.

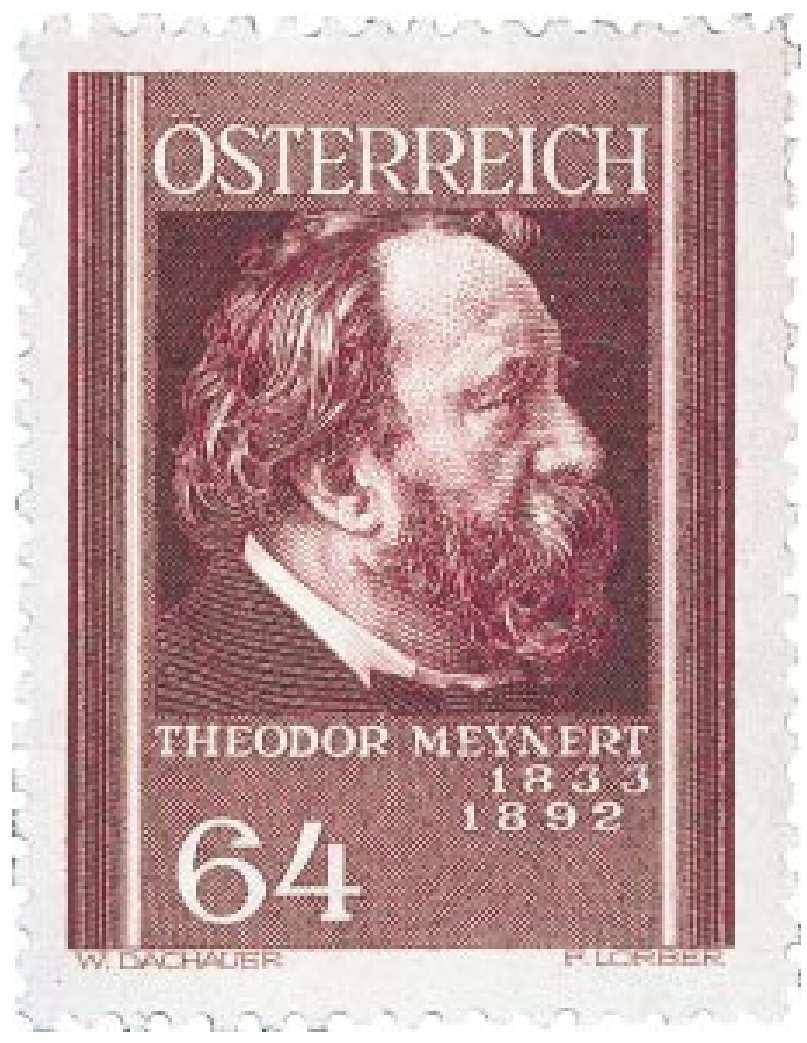

His portrait appears on a set of Austrian postage stamps issued in 1937 to honour famous Austrian physicians (Stanley Gibbons 823, Scott B164).

L F HAAS 